\title{
Ion-Beam Driven Shock Loading Device for Compaction
}

\author{
M. Tsuchida, T. Aizawa and K. Horioka* \\ Department of Metallurgy, University of Tokyo, Tokyo 113, Japan \\ * Department of Energy Science, Tokyo Institute of Technology, Nagatsuda, Kanagawa, Japan
}

\begin{abstract}
Ion-beam driven shock loading device is proposed to make efficient dynamic compaction of nanocrystal and non-equilibrium powders. Different form the conventional gas-guns and powder fire-guns, the repetitive application of shock pressure pulse to targeting materials and the fine control of pressure pulse are intrinsic features to the present approach. Since the generated plasmoid is electro-magnetically accelerated to launch the flyer, no limitation to flyer velocity is present. The laser doppler velocimetry is used to make real time measurement of copper flyer velocity. The efficiency is estimated to be about $6 \%$ for conversion from the accumulated energy in the condenser bank to the kinetic energy of a flyer.

[ion beam, shock wave, powder compaction, non-equilibrium phase, recovery]
\end{abstract}

\section{Introduction}

Three methods are well-known and have been used to generate the shock pressure by acceleration of high density plasma or particles: Intense ion beams [1], Plasma gun [2] and Rail gun [3]. The first candidate has space charge limit so that sufficient current density can never be obtained for its utilization. The second method only utilizes relatively low density plasma with the order of $10^{13} \mathrm{~cm}^{-3}$, so that sufficient number of particles can never be accelerated for generation of shock pressure. The final approach has its limit to speed realized in experiment and often suffers from severe erosion of rails. Considering that repetitive application of shock pressure pulse is indispensable for various shock treatments of materials, new shock loading concept is awaited to propel the shock physics and chemistry by using the high density plasma.

The phased implosion method $[4,5,6]$ is preferable to acceleration of plasma and particles since both the implosion or the pinch time, and their scheduling can be precisely controlled. In our developing method, the plasma density can be compressed to the order of $10^{18}$ to $10^{19} \mathrm{~cm}^{-3}$, and high density plasma can be accelerated by zippering together with axial shock pressure. This acceleration is converted to the impact energy to launch the flyer. Through the previous works, it was experimentally demonstrated that the high energy plasma flow can be electro-magnetically driven by the scheduled capillary Z-pinch. As discussed in Refs. $[5,6,7]$, both the ion velocity and its current density can be evaluated: the estimated value of ion speed from the plasma measurement reaches to $7 \times 10^{7} \mathrm{~cm} / \mathrm{s}$ corresponding to $70-100$ $\mathrm{keV}$ for $\mathrm{Ar}$.

In the present paper, the laser doppler velocimetry, or, VISAR is used to measure the copper flyer velocity for investigation of the relationship between the accelerated plasma and the generated shock pressure. The energy conversion efficiency is estimated to be about $6 \%$ form the accumulated energy in the condenser bank to the kinetic energy of a flyer. Next, copper films and bismuth powders are shot by $\mathrm{Cu}$-flyer to make design of the capsule for recovery testing. Finally, Al-based nanocrystal powders are employed for dynamic compaction through the present shock loading device.

\section{Principle for ion-beam driven shock loading}

As suggested in Refs. $[4,5,6]$, the time response of the $z$ pinched plasma can be estimated by using the snow plow-out model. Considering that the plasma in the cylindrical sheath with the initial radius $r_{0}$ is z-pinched by coaxial discharge, the pinch time $\tau_{\mathrm{p}}$ is approximated by $\tau_{\mathrm{p}}=\mathrm{r}_{0}^{2} / \mathrm{I}_{0}$, where $\mathrm{I}_{0}$ is the initial discharge current. From this equation, it is found that 1) the rapid compaction drives a plasma of small radius with high current level in pinching time, and 2) the pinch time is controllable by varying the initial sheath channel radius. Hence, the control of pinch time by slightly shaping the discharge wall has a potential to accelerate the plasmoid by the scheduled pinching both in the radial and the axial directions. This fundamental concept is schematically depicted in Fig. 1.

High current fast z-discharge with the order of $10^{5} \mathrm{~A}$ leads to generation of an azimuthal magnetic field $\mathrm{B}_{\theta}$ enough to accelerate the plasma radially inward. Due to accumulation and compression of the plasma up to GPa level by the snow plow-out effect, a high density plasmoid is formed at the discharge axis.

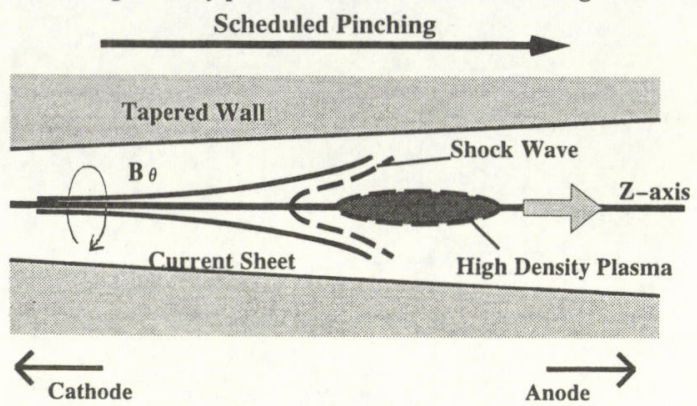

Fig. 1. A concept of ion-beam driven shock loading device. 
The time delay for arrival of this plasmoid is controlled to the pinching process along the discharge axis. To be remembered, in addition to the inward pinching by $\mathrm{B}_{\theta}$, the magnetic flux gradient $\mathrm{Bz}$ is also generated in the driving direction by the phased pinching. Then, the generated plasma can be radially compressed by the self- $\mathrm{B}_{\theta}$ and axially driven by $\mathrm{Bz}$, simultaneously. When the plasma implosion time is exactly phase-matched to the plasmoid transit time, a significant part of the input energy can be converted to the kinetic energy to drive the plasma in the axial direction.

\section{Ion-beam driven shock loading device}

A fast pulse generator LIMAY-I was used to drive the capillary discharge. A high voltage pulse was switched on the electrode after weak pre-ionization discharge. Under this condition, the load current of $80 \mathrm{kA}$ with a rise time of $20 \mathrm{~ns}$ can be driven from the $3 \mathrm{ohm} \mathrm{-} 70 \mathrm{~ns}$ pulse forming line through a pre-pulse suppression SF6 switch. A schematic diagram of the present setup is depicted in Fig. 2. The capillary of $100 \mathrm{~mm}$ length has a thin conical wall, the inlet and the exit diameters of which were 4 $\mathrm{mm}$ and $8 \mathrm{~mm}$, respectively. This capillary was slightly tapered to implode sequentially and to contract the exit plasma within the pulse width $(70 \mathrm{~ns})$ of the discharge in the order of $10^{5} \mathrm{~A}$. Under this situation, the plasma is thought to move axially in a leakystream-tube mode without occurrence of discharge instability.

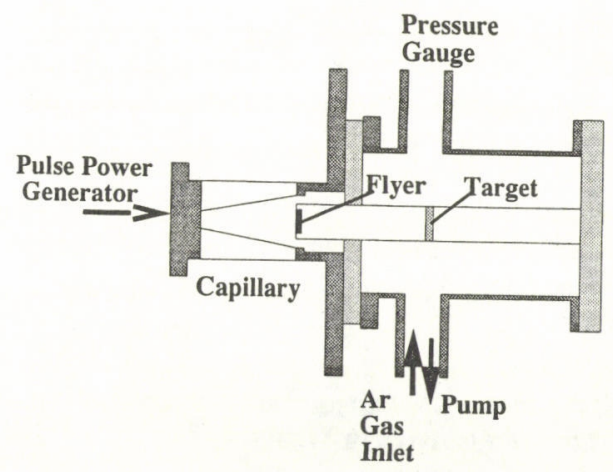

Fig. 2. Experimental set-up for plasma characterization.

The initial static densities of the inert gas were in the order of $10^{17} \mathrm{~cm}^{-3}$, and the background pressure of the time-of-flight (TOF) chamber was kept below $1.33 \times 10^{-1} \mathrm{~Pa}\left(10^{-3} \mathrm{mmHg}\right)$ by the differential pumping. Looking into typical TOF wave forms of the plasma flux with the load current signal, the peak of the plasma signal was found to be shifted in the function of distance and amount of gas loading. When the initial filling pressure was set to be $100 \mathrm{~Pa}$ in $\mathrm{Ar}$, the drift velocity of the plasma became about 7 $x 10^{7} \mathrm{~cm} / \mathrm{s}$. In other words, Ar atoms can travel with the kinetic energy of about $70 \mathrm{keV}$. The measured current density was 200
$\mathrm{A} / \mathrm{cm}^{2}$. In order to check whether the high energy plasma might never be caused by the z-discharge instability, the polarity of the pulse power generator was inverted [5,6]. No change of the plasma pulse profile proves to be a proof that the high energy plasmoid should be purely electro-magnetically accelerated but not by the anormalous electric fields.

\section{Experimental results}

The acceleration test of a mini-flyer was performed by varying the accumulated energy in the condenser bank. Al-based nanocrystal alloy was used to make shock compaction.

\section{1 Real time measurement of flyer velocity}

In order to experimentally demonstrate that the present ionbeam driven shock loading device should have enough controllability to vary the flyer velocity just like the conventional gun facilities, the flyer velocity was measured in real time by using the laser doppler velocimeter. A thin copper metal plate with the thickness of $0.1 \mathrm{~mm}$, the diameter of $5 \mathrm{~mm}$ and the purity of 99 . $9 \%$ was placed at the exit of the capillary tube. Figure 3 depicts a

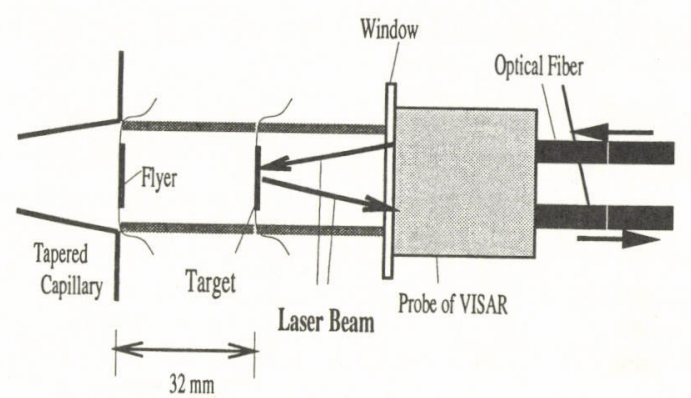

Fig. 3. Schematic view for real time measurement of flyer velocity.

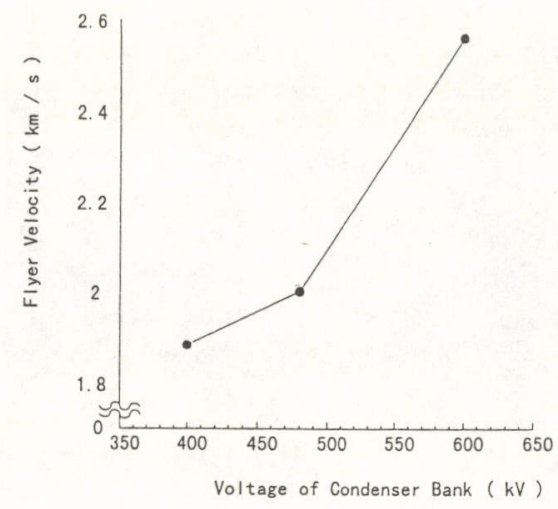

Fig. 4. Relationship between the flyer velocity and the accumulated energy in the condenser bank. 
schematic view of testing: since laser is injected on the flat surface of a flyer, the terminate velocity of the flyer can be directly measured by the laser dopper velocimeter. The charging energy $E_{\mathrm{g}}$ accumulated in the condenser bank was varied to control the flyer velocity $V_{f}$. Variation of $V_{f}$, or, the kinetic energy $E_{f}$ of a flyer with increasing $\mathrm{E}_{\mathrm{g}}$ was depicted in Fig. 4. When $\mathrm{E}_{\mathrm{g}}=1.1$ $\mathrm{kJ}$, the flyer was accelerated up to $2.5 \mathrm{~km} / \mathrm{s}$, and $\mathrm{E}_{\mathrm{f}}$ became about $60 \mathrm{~J}$. This implies that about $6 \%$ of accumulated energy in the condenser bank can be utilized as a kinetic energy to drive a flyer.

\section{2 Cold shock compaction of nanocrystal alloys}

Al-based nanocrystal alloy was employed as a targeting material for cold compaction. Figure 5 shows a typical XRD diagram of this powder; a significant broadening of peaks to constituent elements indicates that this material has nanocrystal structure. The present system was used for cold compaction of this alloy powders into a bulk material. Figure 6 shows the cross-sectional view of the recovered sample. Although the Al-based nanocrystal alloy powders have higher strength over $1 \mathrm{GPa}$ so that the conventional methods are difficult to densify them into a bulk material, they can be compacted in cold by the present method.

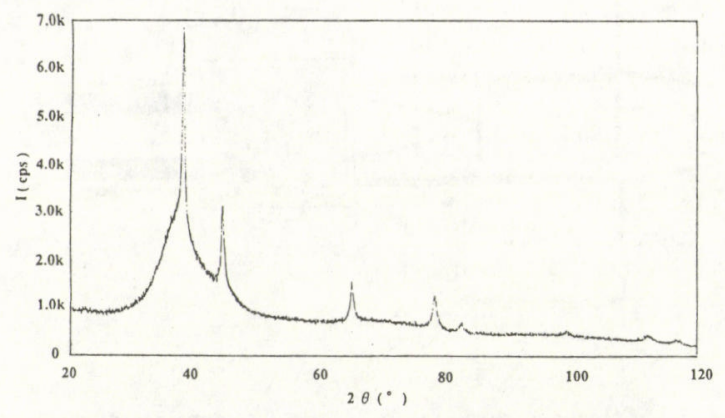

Fig. 5. Typical XRD profile of nano-crystal alloy powders.
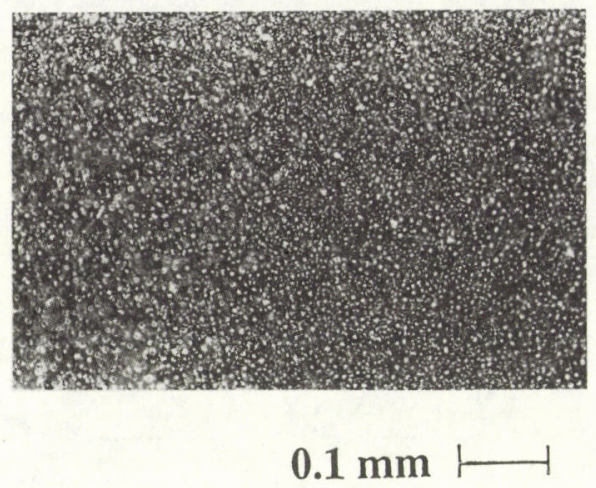

Fig. 6. Cross-sectional view of the recovered sample $\left(V_{f}=2.5\right.$ $\mathrm{km} / \mathrm{s})$.

\section{Discussion}

The ion-beam driven shock loading device has no limits on the flyer velocity in principle since high density plasmoid is electromagnetically driven as demonstrated in this system. Use of 1 to 2 $\mathrm{kJ}$ as an input energy enables us to accelerate the flyer velocity up to 2.5 to $5 \mathrm{~km} / \mathrm{s}$. To be noted is a high efficiency of energy conversion from the input energy to the kinetic energy of a flyer. This proves to be a reliable base to insist that the ion-beam driven shock loading device should be useful to make repetitive shock loading tests and to perform successful recovery testings by shock compaction.

A successful cold compaction of Al-based nanocrystal alloy powders must be a good example to utilize the present shock loading device as a new tool of materials processing without diminishing the non-equilibrium phase and structure in powders. Different from the conventional recovery tests by using gas/fire guns, the present method enables us to shorten the interval time between recovery tests less than 5 to 10 minutes.

\section{Conclusion}

The capsule design suitable to the present recovery test is still necessary to be from cracking in the sample. Simple sample configuration is planned to this cold shock compaction. Our developed ion-beam driven shock loading device is thought to be suitable to shock recovery experiments even subjected to the external fields: hot compaction, cold/hot compaction in the externally applied magnetic field, and so forth.

Furthermore, high efficient shock loading by the present system must be attractive to high velocity shock physics and shock chemistry in the range over $10 \mathrm{~km} / \mathrm{s}$.

\section{Acknowledgment}

This study is financially supported in part by the Grand-in-Aid from Japan Atomic Energy Research Institute.

\section{References}

[1] K. Baumung, J. Singer, S.V. Razorebov and A.V. Utkin: Shock compression of condensed matter. (1995) 1015.

[2] S. Humphries, jr., R.J.M. Anderson, J.R. Freeman and J.B. Greenly: Rev. Sci. Instrum. 52 (2) (1981) 162.

[3] D.A. Tidman and S.A. Goldstein: J. Appl. Physc. 51 (4) (1980) 1975.

[4] K. Horioka, H. Ishikawa, M. Nakajima, T. Hosokai, T. Aizawa and M. Tsuchida: Proc. NIFS (1997) (in press).

[5] K. Horioka, M. Nakajima, T. Aizawa and M. Tsuchida: Proc. $4^{\text {th }}$ Int. Conf. Dense Z-pinches. (1997) (in press).

[6] K. Horioka, T. Aizawa and M. Tsuchida: Shock compression of condensed matter. (1997) (to be published).

[7] M. Tsuchida, T. Aizawa and K. Horioka: J. Materials Processing. (1997) (to be published). 